\title{
DERMATOSIS PROFESIONALES EN CUIDADORES DE ANCIANOS
}

\author{
LUCÍA BARCHINO ORTIZ, ENRIQUE CABRERA FERNÁNDEZ, GREGORIO MORENO MANZANO, \\ FELIPE HERAS MENDAZA Y LUIS CONDE-SALAZAR GÓMEZ
}

\author{
Servicio de Dermatología Laboral. Escuela Nacional de Medicina y Seguridad del Trabajo. \\ Instituto de Salud Carlos III. Madrid. España.
}

\section{RESUMEN}

El progresivo envejecimiento de la población está provocando el desarrollo como grupo profesional de los cuidadores profesionales de ancianos, los auxiliares de geriatría. Puede considerarse un grupo de riesgo para el desarrollo de dermatosis ocupacional debido a las características del trabajo que desempeñan, con una regular exposición a irritantes cutáneos y a trabajo húmedo. Los estudios centrados en la patología cutánea de este colectivo son escasos.

Objetivo: El objetivo de este estudio es describir y analizar las características y los diagnósticos clínicos dermatológicos de los pacientes que trabajan como cuidadores de ancianos valorados en nuestro servicio.

Material y métodos: Se ha llevado a cabo un estudio observacional retrospectivo de los datos de 41 pacientes cuidadores de ancianos estudiados en nuestro servicio durante un periodo de 11 años.

Resultados: Encontramos un predominio total de mujeres. El lugar de afectación más frecuente fueron las manos. Los diagnósticos más frecuentemente establecidos fueron: Eczema endógeno (39,6\%), dermatitis de contacto irritativa (DCI) $(27,1 \%)$, dermatitis de contacto alérgica (DCA) (12,5\%), Sin lesiones $(10,4 \%)$, urticaria de contacto al látex $(4,2 \%)$ y otros.

Conclusiones: El colectivo de cuidadores de ancianos es un grupo en aumento y de gran importancia social. Al desempeñar una labor que implica un trabajo húmedo se debe considerar un grupo de riesgo para el desarrollo de dermatosis profesionales. En nuestro estudio destaca que el diagnóstico de eczema endógeno, representado por la dermatitis atópica, sea el más frecuente, explicándose esto porque una irritación provocada por un trabajo húmedo y la oclusión pueden desencadenar un eczema en el individuo con un fondo atópico, que en otras condiciones se habría mantenido latente. Son necesarios futuros estudios más extensos en este sector profesional.

\section{PALABRAS CLAVES}

Dermatosis ocupacionales, cuidadores de ancianos, auxiliares de geriatría, trabajo húmedo.

\section{ABSTRACT}

The increasing proportion of elderly citizens worldwide is leading to the development of a new professional group of people who are specialized in elderly personal care. They may have an increased risk of occupational skin damage due to the characteristics of their work that is often associated with regular exposure to skin irritants and constant wetwork. Skin disease studies within this group are rare.

Aims: The aim of this study was to describe and analyse the characteristics and dermatologic diagnosis of the patients that work taking care of elderly people studied in our department.

Methods: We studied retrospectively all 41 patients who work as elder care-taker who attended our department in a 11-year period.

Results: We found an absolute female predominance. The most common location affected was the hands. Of the skin diseases clinically diagnosed, endogenous eczema showed the highest prevalence $(39,6 \%)$ followed by irritant contact dermatitis (ICD) (27,1\%), allergic contact dermatitis (ACD) $(12,5 \%)$, asymptomatic $(10,4 \%)$, allergy to natural rubber latex (NRL) $(4,2 \%)$ and others.

Conclusions: Elderly care-taker workers are emerging and they have a great social importance. They are at increased risk of occupational skin disease due to contact with several substances and wet work. In our study, endogenous eczema, represented by atopic dermatitis, is the most important diagnosis. It could be concluded that irritants, occlusion and wet work favoured the development of dermatitis in vulnerable patients that would be remained asymptomatic avoiding this work. New further studies are needed.

\section{KEY WORDS}

Occupational skin disease, elderly care, geriatric nursing assistant, elderly care-taker worker, wet work. 


\section{INTRODUCCIÓN}

Las dermatosis constituyen una de las enfermedades profesionales más frecuentes en los países industrializados siendo, en algunos de ellos, las primeras enfermedades profesionales notificadas y más del 30\% de las que requieren compensación económica. Las más importantes son las dermatitis de contacto, ya que representan el $90-95 \%$ de los casos. La forma de presentación más frecuente, y por tanto uno de los principales problemas valorados en dermatología ocupacional, es el eczema de manos. $1,2,3,4,5,6,7$

Es difícil calcular la incidencia de las dermatosis profesionales. En Europa se estima que la incidencia media oscila entre 0,5-1,9 casos por 1000 trabajadores por año, pero la comparación entre países es compleja y suelen ser cifras infraestimadas, debido a que los registros nacionales que proporcionan los datos se basan en la notificación de casos, que por lo general se realiza cuando se declara la baja o la incapacidad. ${ }^{2,4,5}$

El desarrollo de la dermatitis de contacto profesional, tanto irritativa como alérgica, está determinada por la combinación de factores de susceptibilidad individual (por ejemplo, la diátesis atópica) y la exposición a factores exógenos. Diversos estudios han señalado como factor de riesgo fundamental la exposición a irritantes y han reconocido como el más importante el trabajo húmedo, que llega incluso a duplicar el riesgo de padecer eczema de manos. ${ }^{1,2,3,6,8,9}$ El trabajo húmedo supone una exposición repetida a agua y jabones unida a la oclusión producida por los guantes. Es decir, se trata de un trabajo que produce maceración de la piel, y en el que el mojado y secado repetido dan lugar a descamación y fisuración que facilitan la penetración, tanto de alergenos como de agentes irritantes. ${ }^{2,4,5}$ Según la legislación alemana, el trabajo húmedo se define como aquél en el que los trabajadores exponen sus manos a líquidos o portan guantes oclusivos durante más de 2 horas diarias o llevan a cabo un lavado de manos intenso y frecuente. ${ }^{2,5}$

Por tanto, las profesiones que implican trabajo húmedo, en las que se incluye el personal sanitario, pueden ser consideradas de alto riesgo para el desarrollo de dermatosis profesional, estimándose una prevalencia media en estos trabajadores sanitarios que oscila entre el 17 y $20 \%$ según diversos estudios de la literatura. ${ }^{8,9,10}$ En un estudio poblacional realizado en la región de Baviera para identificar los grupos de mayor riesgo, se encontró que la dermatitis de contacto irritativa de manos era la más frecuente entre las dermatosis ocupacionales y que las tasas de incidencia global eran de 4,5 por 10.000 trabajadores por año para las dermatitis de contacto irritativa (DCI) y 4,1 para la dermatitis de contacto alérgica (DCA), mientras que entre los trabajadores sanitarios éstas eran de 4 y 3,7 respectivamente ${ }^{6}$. Sin embargo, existen variaciones según la especialidad y el tipo de funciones, siendo el colectivo de enfermería y auxiliares de enfermería el de mayor riesgo. ${ }^{6,8}$

Por otra parte, queremos resaltar otro hecho importante que está provocando un gran cambio sociodemográfico y laboral en nuestra sociedad: El envejecimiento de la población. El aumento de la esperanza de vida, junto con la disminución de las tasas de fecundidad, están conduciendo a un progresivo crecimiento de la población anciana en todo el mundo y, de manera más importante, en los países desarrollados. ${ }^{11,12}$

En España, las proyecciones de población calculadas a partir del Censo de 2001 reflejan una clara tendencia creciente de la proporción de población de 65 o más años que prácticamente duplicará su peso relativo al pasar del $16.7 \%$ de la población (6.958.516 personas) en 2001, al 30,8\% (16.394.839 personas) en el año $2050 .^{13}$

Se trata de un grupo poblacional que en muchas ocasiones necesita cuidados, por lo que ante este proceso imparable de envejecimiento poblacional, la atención y cuidado de las personas mayores se ha convertido en un reto y una necesidad importante de la sociedad y el sistema sanitario. ${ }^{11}$

Para atender esta demanda, los servicios residenciales están aumentando de manera espectacular. En España, en el periodo de tiempo comprendido entre 1994 y 2004, el número de centros residenciales (públicos y privados registrados) ha aumentado de 2.702 a 4.888 , con un aumento total de plazas de 67.334. ${ }^{12}$ De esto se puede deducir el importante potencial del sector como empleador dentro de los servicios sanitarios y que pueda considerarse una profesión en auge con expectativas de trabajo futuro. (Por ejemplo, en la Comunidad de Madrid se crearon 6.640 puestos de trabajo dentro del Servicio de Atención Residencial en el año 2003). ${ }^{12}$

Por ello queremos hacer hincapié en un grupo profesional emergente como es el de los cuidadores profesionales de ancianos, los auxiliares de geriatría. Hay que destacar el hecho de que es un subgrupo particular dentro del sector sanitario, ya que por una parte atiende a un grupo de enfermos con altísimo nivel de dependencia del cuidador para las activida- 
des de la vida diaria, y por otra lleva a cabo un trabajo muy variado, con tareas no bien definidas, que incluyen un constante trabajo húmedo, como son el lavado de los pacientes, su aseo diario, limpieza de camas y manejo de múltiples productos; unido al propio lavado repetido de manos y al uso de guantes para la mayoría de las actividades. ${ }^{14}$ Todo ello conllevaría un alto potencial de riesgo de dermatosis profesionales, tanto alérgicas como irritativas, en este sector. Aunque se han llevado a cabo algunos estudios centrados en el análisis de la patología cutánea de los profesionales que desempeñan su trabajo en residencias de ancianos en países asiáticos y Australia $^{14,15,16,17}$ las investigaciones en otros lugares, incluyendo España, son inexistentes.

Nuestro objetivo es describir y analizar las características clínicas y ocupacionales de los pacientes que trabajan como cuidadores de ancianos que acuden a nuestro servicio y llamar la atención sobre la creciente importancia de las dermatosis ocupacionales en este grupo profesional.

\section{MATERIAL Y MÉTODOS}

Hemos llevado a cabo un estudio observacional retrospectivo basado en el análisis de datos obtenidos de las historias clínicas de nuestra base de datos de todos los pacientes pertenecientes al grupo profesional de cuidadores de ancianos (auxiliares de geriatría) valorados en nuestro departamento por problemas cutáneos en el periodo de tiempo comprendido entre el 1 de Enero de 1995 y el 31 de diciembre de 2005.

En las historias clínicas se incluía una anamnesis completa que consta de datos epidemiológicos (edad, sexo), antecedentes personales (enfermedades previas e historia de atopia, haciendo hincapié en intolerancia a metales, rinoconjuntivitis polínica y dermatitis atópica), historia laboral (trabajo actual y previos, duración de cada uno, productos utilizados y medidas protectoras usadas...) y síntomas (tipo, localización de las lesiones, duración, relación con posibles causas...) junto con la exploración física, las pruebas realizadas, el diagnóstico establecido y las recomendaciones dadas al paciente.

Se realizaron pruebas epicutáneas en aquellos casos que tenían una historia clínica sugerente de dermatosis profesional de probable origen alérgico. En todos estos casos se parcheó la serie estándar del Grupo Español de Investigación en Dermatitis de Contacto (GEIDC) y se añadieron otras baterías especiales de alergenos (suministradas por Chemotechnique Diagnostics) si se consideraba necesario según la patología que presentaban (gomas, metales, fragancias, cosméticos y productos propios). Asimismo, en 20 pacientes $(48,8 \%)$ se realizó el prick test de sensibilidad al látex.

Estas pruebas se aplicaron en la parte superior de la espalda, se fijaron con tela adhesiva y fueron retiradas a los dos días. Las lecturas se efectuaron al segundo y cuarto día utilizando para ello el sistema de valoración de "cruces" $(+,++$ y +++), tal y como recomienda el Internacional Contact Dermatitis Research Group (ICDRG), determinándose posteriormente su relevancia en cada caso.

\section{RESULTADOS}

El grupo profesional de cuidadores de ancianos está formado por 41 pacientes, que constituyen el $0,52 \%$ del total de 7.914 pacientes atendidos en nuestro Servicio en este periodo de 11 años. Estos pacientes se distribuyen a lo largo de los años según la gráfica de la figura 1.

Figura 1. Porcentaje de auxiliares de geriatría valorados anualmente en el Servicio de Dermatología Laboral de la ENMT.

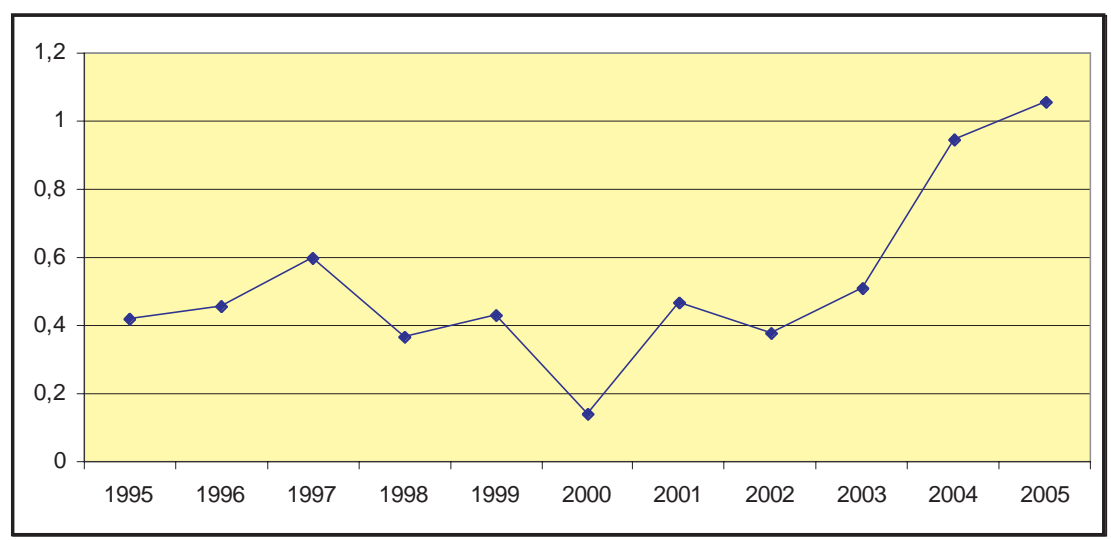


Del total de 41 casos atendidos, el $100 \%$ eran mujeres y la edad media era de 33,12 años con un rango que oscila entre los 19 y los 56 años.

En cuanto a los antecedentes personales, se trata de una muestra en la que el 43,9\% (18 pacientes) presentan estigmas de diátesis atópica frente al $56,1 \%$ (23 pacientes) que no los tienen. Un $56,1 \%$ de ellos refiere intolerancia a metales, un $34,1 \%$ son polínicos (rinoconjuntivitis y/o asma alérgicas) y un 7,3\% sufre dermatitis atópica.

La totalidad de las pacientes trabajaba como auxiliar de enfermería en el cuidado de ancianos (auxiliar de geriatría). La mayoría (92,7\%) lo hacían en residencias geriátricas, mientras que un $4,9 \%$ y un 2,4\% lo hacían en hospitales geriátricos y en domicilios particulares respectivamente. El tiempo medio en el actual puesto de trabajo fue de 70,6 meses, con un rango de 0,5 a 432 meses y una mediana de 48 meses (4 años). Excluyendo 4 casos $(9,75 \%)$ en los que se desconoce el tiempo, 22 casos llevaban 4 o menos años en dicha ocupación, lo que supone un 53,7 \% de los mismos. En 22 casos
(53,7\%) nunca habían desarrollado un trabajo previo, a diferencia de los 19 (46,3\%) que sí lo habían hecho.

El 100\% de los pacientes refería usar guantes como medida protectora, siendo los más utilizados los de látex-goma (82,9\%), seguidos de los de vinilo $(34,15 \%)$, algodón (21,95\%) y plástico $(9,75 \%)$. Un total de 10 pacientes $(24,39 \%)$ relaciona su patología con el uso de guantes.

En cuanto a la localización de la clínica, el lugar más afectado son las manos, con 29 pacientes afectados (70,7\%), 27 de manera exclusiva y 2 concomitantemente a los pies, seguido de afectación diseminada (14,6\%), cara $(7,3 \%)$, miembros $(4,9 \%)$ y exclusivamente pies (2,4\%).). [Figura 2].

El tiempo de evolución de las lesiones era de menos de 1 mes en 2 casos (4,9\%), entre 1 y 6 meses en 15 casos (36,6\%), entre 6 meses y 1 año en 11 casos (26,8\%) y de más de 1 año en 13 casos (31,7\%). [Figura 3].

Figura 2. Localización de la clínica.

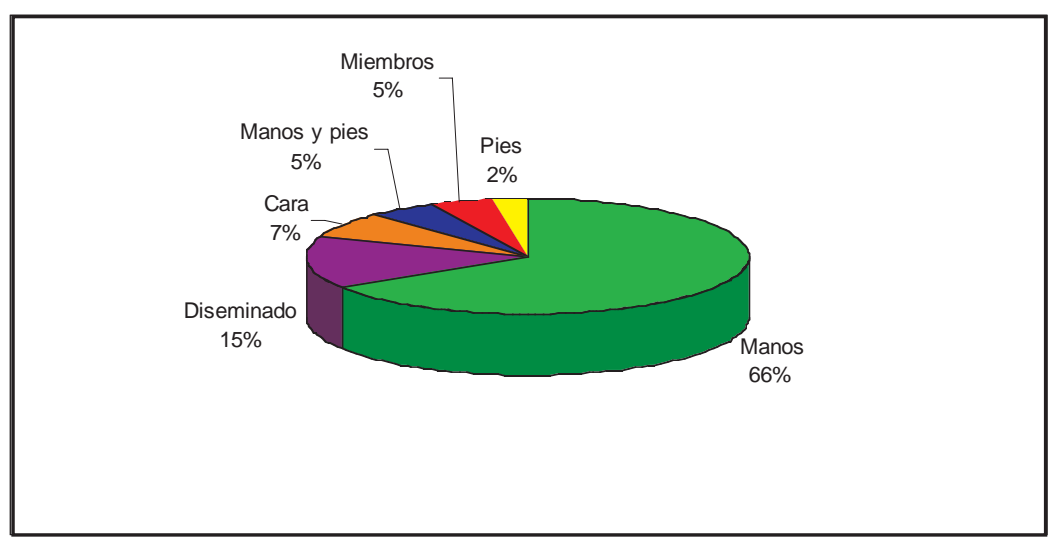

Figura 3. Tiempo de evolución de la sintomatología.

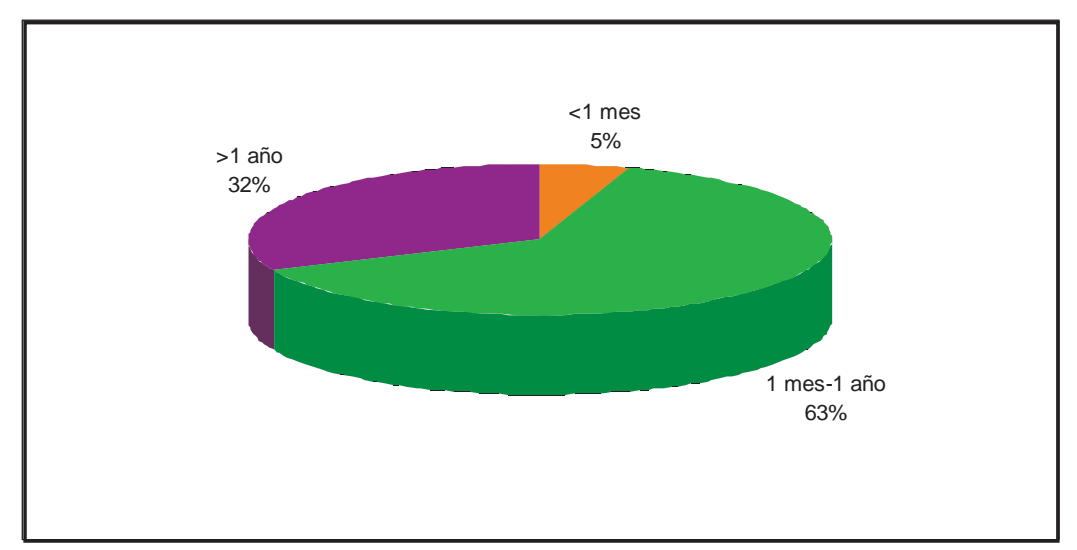


Las pruebas epicutáneas estándar fueron realizadas en 34 de los 41 casos (83\%), ya que 3 personas no completaron el estudio y en el resto (4 personas) no se consideró necesario realizar dichas pruebas. De estos 34 casos, 22 mostraron al menos 1 positividad, representando esto un 64,7\% de los parcheados, mientras que en 12 (29,3\%) no se observó ninguna [Figura 4]. Dentro de las 30 baterías adicionales sólo se encontraron sensibilizaciones a algún componente de las mismas en 12 casos, y sólo 1 en los 9 pacientes a los que se les parchearon productos propios.

Figura 4. Porcentajes de los resultados de las pruebas epicutáneas

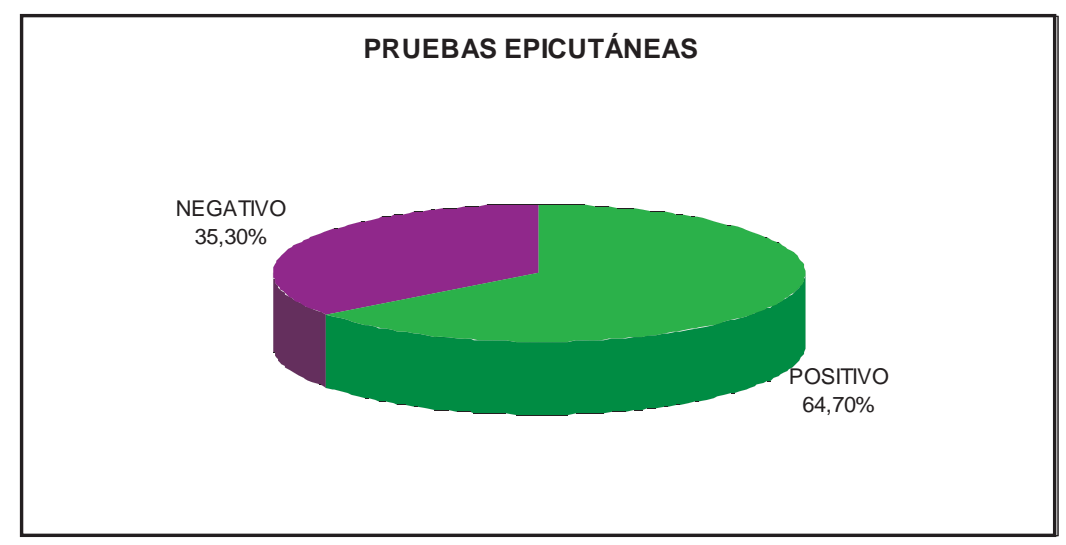

En cuanto a las sensibilizaciones más frecuentemente encontradas en estos pacientes destacan el sulfato de níquel (15 casos), cloruro de cobalto (7 casos y siempre asociado a níquel o cromo), dicromato potásico (4 casos), mezcla de fragancias (4 casos), parafenilendiamina (3 casos), mezcla de tiuram (3 casos), paladio (3 casos), Katon CG (2 casos), Quaternium (2 casos), tetraetiltiuram disulfuro (TETD, 2 casos), isoeugenol (2 casos) y Tiomersal (2 casos), así como 1 sólo caso de neomicina, benzocaína, resinas epoxi, bálsamo de Perú, etilen- diamina, paraterbutilfenolformaldehído (PTBF), mezcla de carbas, tetrametiltiuram monosulfuro (TMTM), tetrametiltiuram disulfuro (TMTD), dipentametilentiuram disulfuro (PTD), cadmio, oro, eugenol, geraniol, ptoluendiamina, paraaminoazobenceno y zapatos propios.

Sin embargo, las relevancias de las diferentes sensibilizaciones son considerablemente menores, tal y como se detallan en la gráfica de la figura 5.

Figura 5. Tabla comparativa del número de positividades y su relevancia con la clínica.

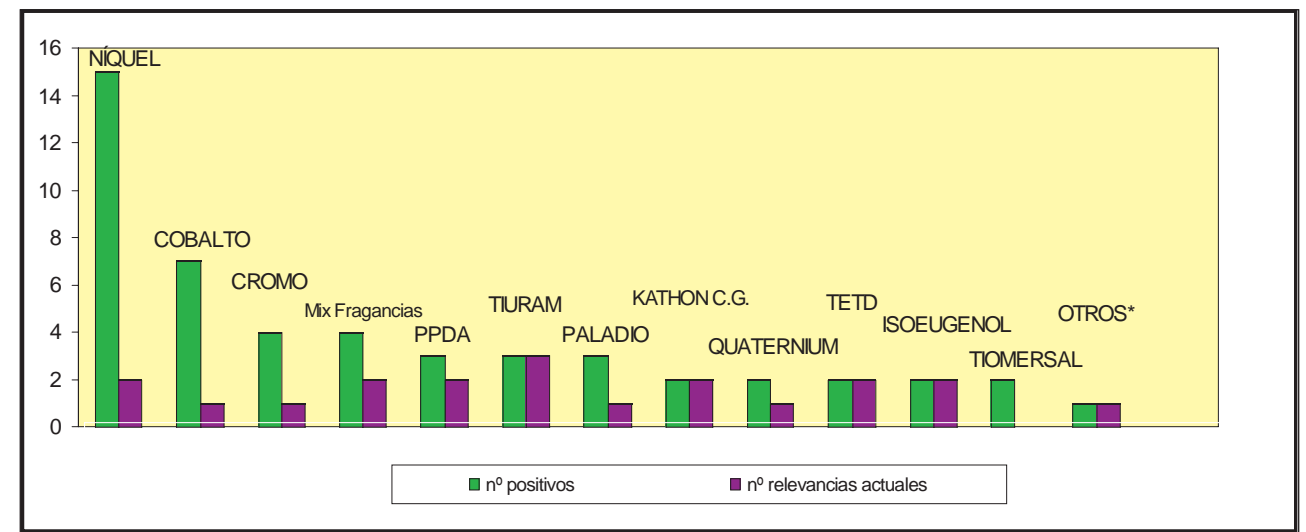

* Otros: Bálsamo de Perú, etilendiamina, mezcla de carbas, TMTD, PTD, Eugenol, Geraniol, Ptoluendiamina, Paraaminobenceno, zapatos. 
Por orden de mayor a menor frecuencia, los diagnósticos establecidos en este grupo fueron: Eczema de tipo endógeno en 19 casos (39,6\%), que correspondieron a eczema dishidrótico (8 casos), eczema en placas (4 casos), dermatitis atópica (5 casos) y dermatitis seborreica (2 casos); seguido de dermatitis de contacto irritativa (DCI) en 13 casos (27,1\%); dermatitis de contacto alérgica (DCA) en 6 casos (12,5\%); en 5 casos los pacientes no presentaban lesiones y por tanto se les consideró sin patología actual (10,4\%); urticaria de contacto al látex en 2 casos (4,2\%); y dermatitis psoriasiforme, dermatitis residual y sensibilidad al látex en 1 caso respectivamente. Agrupando estas categorías podemos apreciar globalmente los diagnósticos en el gráfico de la figura 6.

Figura 6. Diagnósticos principales.

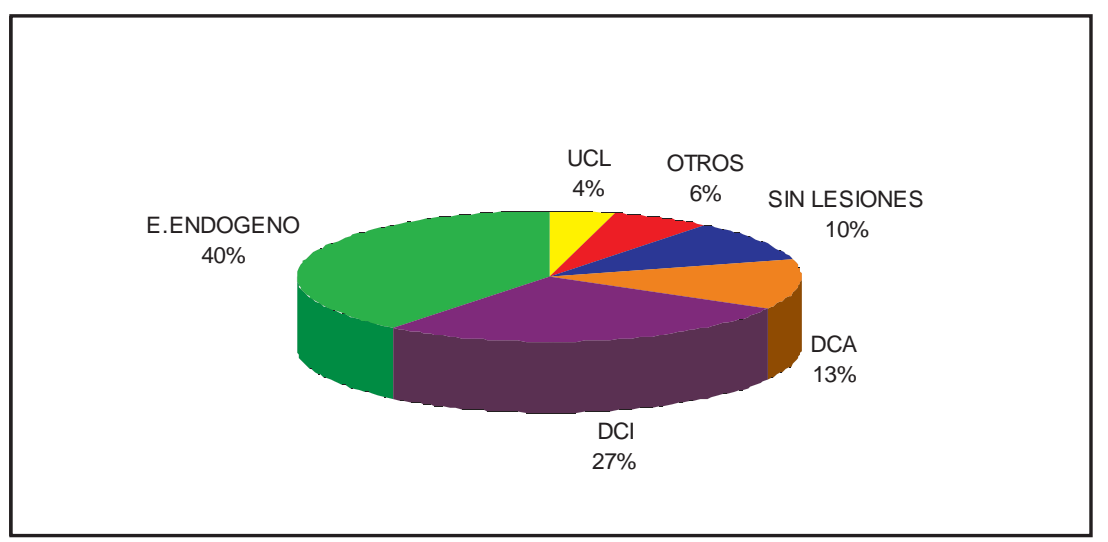

E. ENDÓGENO = Eczema endógeno; UCL = Urticaria de contacto al látex; DCI = Dermatitis de contacto irritativa; DCA = Dermatitis de contacto alérgica.

Las sensibilidades relacionadas con los casos de dermatitis de contacto alérgica son variadas. Hay 2 casos relacionados con alergia a componentes de las gomas (mezcla de Tiuram, mezcla de carbas, TMTM, TMTD, TETD, PTD), 2 casos relacionados con alergia a las fragancias (mezcla de fragancias, bálsamo de Perú, eugenol, isoeugenol y geraniol) y 1 caso de alergia al cromo como componente de los zapatos y otro con alergia a ptoluendiamina, paraaminoazobenceno y PPDA.

\section{DISCUSIÓN}

El progresivo envejecimiento de la población está provocando el desarrollo de un nuevo grupo profesional cada vez más numeroso, el de los cuidadores de ancianos. Es una profesión en auge y de futuro, ya que para responder a la demanda surgida en la sociedad, los servicios de atención al mayor, tanto a nivel público (residencias de ancianos, teleasistencia, hospitales geriátricos...), como privado (ancianos que viven solos o con su familia y precisan cuidados) requieren cada vez un mayor número de personas dedicadas a esta tarea.
Por tanto, se trata de un colectivo amplio, no bien caracterizado aún, con un perfil de trabajo particular que se distingue por su gran variedad, ya que al atender a un grupo de enfermos con altísimo nivel de dependencia para las actividades de la vida diaria, llevan a cabo múltiples tareas no bien definidas. Éstas, a modo de ejemplo, incluirían desde la atención personal (aseo diario, lavado de los pacientes en ducha o baño, higiene buco-dental con manejo de prótesis, hidratación y protección de la piel, ayuda a levantado y acostado de la cama, cambio de pañales...), hasta el adiestramiento en actividades de la vida diaria (ayuda a preparar e ingerir alimentos, vestido, afeitado, peinado del cabello e incluso depilación facial), tareas domésticas (hacer camas, cambio de sábanas...), servicio de compañía (ayuda a caminar, a leer...) y atención sanitaria (movilización, cambios posturales, cuidado de heridas administración de medicación oral, prevención de úlceras por decúbito... $)^{9,10}$. De hecho, en un estudio en profesionales de la limpieza en residencias de ancianos fue difícil definir el grupo de estudio, ya que sus funciones de limpieza se mezclaban con otras tareas como el cuidado de ancianos y el trabajo en la coci$n \mathrm{a}^{18}$. Muchas de estas funciones implican tanto una 
constante exposición a trabajo húmedo, como contacto con múltiples irritantes (agua, jabón, detergentes, desinfectantes, antisépticos, guantes de goma, comidas...), potenciales alergenos (gomas de los guantes, conservantes, cosméticos, fragancias, medicaciones tópicas...), labores que producen un daño físico directo en la piel y riesgo de potenciales infecciones ${ }^{14}$, siendo por tanto un grupo que puede considerarse de riesgo para el desarrollo de dermatosis profesional.

Además, hay que considerar que aparte del personal "profesional" que con la formación de auxiliares de enfermería o geriatría atiende a los ancianos en residencias, hospitales o domicilios, existe también un grupo de personas no cualificadas que está realizando estas mismas funciones a otros niveles, fundamentalmente domiciliarios y que quedaría fuera del sistema de notificación de enfermedades profesionales, así como del grupo de pacientes que nosotros hemos valorado. Por tanto, la incidencia real de dermatosis profesional en este tipo de trabajadores podría ser aún mayor.

Centrándonos en nuestro estudio es destacable el porcentaje de mujeres en el grupo, ya que a pesar de que el personal sanitario es mayoritariamente femenino (2/3 del personal son mujeres) y ésto es aún más marcado en especialidades como la enfermería (incluyendo auxiliares) $)^{1,8,19}$ en este caso el predominio es absoluto. Esto se podría relacionar con el hecho de que el cuidado de ancianos se ha considerado siempre como una labor llevada a cabo fundamentalmente por mujeres (madres, hijas, hermanas...) que atendían a los mayores de sus familias.

La edad media del grupo era de 33,12 años y el 46,34\% había desarrollado un trabajo previo. El rango de edad es bastante amplio (de 19 a 56 años) $\mathrm{y}$ aunque no se puede asegurar con certeza, esto podría atribuirse al hecho de que contáramos con dos tipos de trabajadoras diferenciadas: Por una parte jóvenes que comienzan su primer trabajo, y por otra, mujeres mayores con dificultad para encontrar empleo que hallan en este sector su oportunidad. Esto coincidiría en parte con un estudio reciente que compara las alteraciones cutáneas entre las estudiantes de enfermería geriátrica con otras especialidades, en el que además se destaca el hecho de que estas personas, normalmente amas de casa que después de criar a sus hijos comienzan a trabajar, ya se han expuesto a otras ocupaciones de riesgo para dermatosis $^{20}$. Sin embargo, en nuestro estudio también hay mujeres jóvenes, que bien por vocación o bien por garantía de empleo, eligen esta profesión.
En cuanto a la localización de la sintomatología, en nuestra muestra lo más frecuente fue la afectación de las manos, que se confirmó en 29 pacientes, un $70,73 \%$ de los casos. Ésto se explicaría por el hecho de que son la parte más expuesta al contacto con irritantes o alergenos y coincide con los datos existentes acerca de las dermatosis profesionales, en las que el eczema de manos representa aproximadamente el 90\% de los mismos. ${ }^{1,2,4,5,7,19,21,22,23,24,25}$ La prevalencia de la afectación de las manos varía entre distintos estudios, con oscilaciones desde el $48,5 \%$ en sanitarios1 hasta el $80 \%-90 \%{ }^{4,22}$ a nivel general. Es conocido que el riesgo de las profesiones con trabajo húmedo es mayor y diferentes estudios epidemiológicos han identificado estas profesiones, entre las que se encuentran los trabajadores sanitarios, junto a otras como limpiadoras o cocineros, como factor de riesgo de eczema de manos. ${ }^{18,24,26}$

El diagnóstico más frecuentemente establecido fue el de las formas endógenas de eczema (39,6\%), seguidas de la dermatitis de contacto irritativa (27\%) y la dermatitis de contacto alérgica (13\%). Aunque el tamaño de la muestra es pequeño, y por lo tanto las conclusiones podrían estar sesgadas, resulta llamativo que el eczema de tipo endógeno sea lo más frecuente.

Es cierto que todos estos diagnósticos son compatibles con la naturaleza de las labores realizadas por estos profesionales, que como ya se ha señalado son múltiples y variadas pero, sin embargo, en múltiples estudios se considera que el factor de riesgo fundamental para el desarrollo de dermatosis ocupacional es la exposición a irritantes ${ }^{5}$ y en distintas investigaciones se ha señalado la dermatitis de contacto irritativa como la forma más frecuente de dermatosis ocupacional tanto a nivel global como dentro del personal sanitario, ${ }^{21,27}$ incluyendo el sector de enfermería (enfermeras y sus auxiliares) donde se considera que el trabajo húmedo es sin duda el más dañino, además de la actividad con más peso en su labor, habiéndose considerado incluso de mayor importancia que el uso de guantes continuado en el subsector de las residencias de ancianos. ${ }^{23,28}$

Por lo tanto, en este caso, aunque no se puede olvidar que el eczema de contacto es siempre un diagnóstico a tener en cuenta y que hemos encontrado globalmente un $40 \%$ de los pacientes afectados por el mismo y un porcentaje elevado de la variante irritativa, lo primordial es valorar la influencia de los elementos endógenos, representados por la diátesis atópica, en el desarrollo de dermatosis. En nuestra muestra, el 43,9\% (18 pacientes) presentan estigmas de diátesis atópica, refiriendo un 34,14\% síntomas 
de polinosis y habiendo sido diagnosticados previamente de dermatitis atópica un 7,3\%. El porcentaje de diátesis atópica global es alto (aunque los datos publicados en la literatura son variables) pero los casos de eczema endógeno diagnosticados lo son aún más, y sólo 9 de los 19 son personas con diatesis atópica previa recogida en la anamnesis. Aun con las limitaciones de nuestra muestra, podemos explicar esto por el hecho de que personas susceptibles que nunca han desarrollado otra sintomatología y que no lo harían en otras circunstancias, al estar sometidas a este tipo de trabajo, debutan con eczema de manos de tipo endógeno o una mezcla difícil de catalogar entre forma irritativa más endógena. No se ha podido valorar si los pacientes con diátesis atópica desarrollan más frecuentemente dermatosis que los que, no ya que partimos de un grupo sesgado en el que sólo hay 5 pacientes sanos. Tampoco se encontraron diferencias en la edad de debut de los pacientes atópicos (media de 33,5 años en atópicos vs 33,2 años en no atópicos).

Esta es una cuestión algo controvertida, ya que existe una gran variabilidad en la prevalencia de atopia encontrada en diversos estudios, desde un $6,9 \%{ }^{27}$ a un $15-20 \%{ }^{1}$ en personal sanitario, hasta un $45 \%{ }^{24}$ en el estudio llevado a cabo en enfermeras geriátricas. Lo que sí se ha demostrado en la mayoría de los estudios es que la atopia es un factor de riesgo significativo para el desarrollo de dermatosis profesionales al hacer a la persona más susceptible, sobre todo en este tipo de trabajos, a las agresiones externas. De este modo se ha encontrado que el $37 \%$ de los trabajadores con dermatitis atópica desarrolla una dermatosis profesional, siendo esto superior al $20 \%$ de la población general ${ }^{25}$. La historia de atopia multiplica por 3 el riego de desarrollar eczema de manos, tanto en trabajos húmedos como secos ${ }^{1}, \mathrm{y}$ aunque esta asociación sólo se ha demostrado con la dermatitis de contacto irritativa y no con la forma alérgica $^{27}$, lo que es innegable es que muchas veces están interrelacionadas entre ellas, ya que un factor para el desarrollo de esta dermatosis alérgica es la existencia de una alteración cutánea previa como la dermatitis irritativa. ${ }^{27,29}$

También es bastante significativo que aunque se encontraron sensibilizaciones a diversos componentes, la relevancia de las mismas fue considerablemente menor, lo que vuelve a indicar que la mayoría de los casos no corresponden a DCA sino a DCI o empeoramiento de una forma endógena no manifestada hasta la actualidad. En nuestro grupo de cuidadores de ancianos, el sulfato de níquel fue el alergeno más frecuentemente encontrado, seguido de cloruro de cobalto, dicromato potásico, fragan- cias mix, parafenilendiamina y tiuram mix, pero es importante valorar su verdadera relevancia clínica. El más relevante a nivel ocupacional fue el tiuram mix seguido de sulfato de níquel, fragancias mix, parafenilendiamina y clorometilisotiazolinona, con unos porcentajes de relevancia con respecto a las positividades de $100 \%, 13,3 \%, 50 \%, 66,7 \%$, y $100 \%$ respectivamente.

También en otros estudios se encontró baja tasa de relevancia actual. En uno de ellos, sólo se encontró un 29\% de relevancia ocupacional de los resultados positivos a nivel global y un $26 \%$ en los profesionales de la salud. La sensibilización más relevante ocupacionalmente en estos profesionales fue también el tiuram mix, seguida de sulfato de níquel, formaldehído, fragancias, clorometilisotiazolinona, cobalto, parafenildiamina, bálsamo de Perú, mercurio, colofonia, dicromato potásico y otros. ${ }^{30}$ Aunque existen variaciones en los alergenos más frecuentemente implicados en los distintos estudios, siempre destacan los derivados de las gomas, biocidas/conservantes (glutaraldehído y cloruro de benzalconio), medicaciones y sulfato de níquel, entre otros $^{9,27,29}$

El tiuram mix es uno de los aditivos de las gomas y se considera que la mayor parte de los casos de sensibilización son causados por el contacto con guantes de goma. Existen múltiples estudios en profesionales sanitarios donde los componentes de las gomas son unos de los alergenos más frecuentemente implicados (con un $85 \%$ de relevancia de los casos positivos) y por tanto se considera un grupo profesional de riesgo para padecer DAC a este compuesto ${ }^{9,27,29,30}$ También en el caso concreto de las enfermeras de geriatría, un 56\% de las parcheadas mostró sensibilización a alergenos relevantes en el trabajo, estando incluídos los componentes de las gomas, junto a conservantes, vehículo-emulsificantes y fármacos, así como fragancias $(17 \%)^{24}$. Otro estudio ha determinado que en el grupo de enfermeras el alergeno no suele corresponderse con la exposición laboral. ${ }^{29}$

Hay que recordar que el sulfato de níquel, como ya se ha referido previamente, es el sensibilizante más frecuente, pero esta alta tasa de sensibilización no es de origen profesional, sino que se relaciona con el uso de objetos metálicos de bisutería, sobre todo en mujeres. Puede ir unida a sensibilización al cloruro de cobalto, que se encuentra fundamentalmente como contaminante de los productos con níquel. La relevancia ocupacional es de un 13,3\% (similar al 12\% encontrado en la literatura) y podría deberse al uso de distintos objetos metálicos. Tam- 
bién se cree que podría estar influenciado por otros factores como la atopia, el trabajo húmedo e irritantes cutáneos que alterarían la barrera cutánea, haciendo que bajísimas concentraciones provocaran o agravaran una dermatitis. ${ }^{30}$

La mezcla de fragancias tiene una relevancia de un $50 \%$ (33\% en ambiente sanitario global) y se explicaría porque se encuentran en medicamentos y otros productos tópicos, que son muy utilizados tanto en sanitarios como en cuidadores de ancianos en particular. Son muchos los estudios realizados para valorar la importancia laboral de las fragancias, ya que son sustancias ampliamente utilizadas, tanto a nivel particular como laboral, y en algunos trabajos es el contactante más frecuente en la población parcheada, después del níquel ${ }^{31}$. Existen algunos trabajos en los que se ha identificado un riesgo aumentado de sufrir dermatitis de contacto alérgica a las fragancias por parte de los trabajadores sanitarios $(11,7 \% \text { de los hombres y } 10,4 \% \text { de las mujeres })^{32}$. Se cree que es debido a que enfermeras y médicos tienen la necesidad de lavarse las manos regularmente para combatir la extensión de las infecciones y los jabones, soluciones antisépticas y cremas emolientes suelen estar perfumadas. Además, las enfermeras bañan e hidratan a los pacientes exponiéndose a fragancias $^{32}$. Otro trabajo identificó como una de las profesiones de mayor riesgo a las enfermeras geriátricas, debido al uso de productos usualmente perfumados para el lavado y desinfección de manos, junto a la aplicación de productos y medicamentos tópicos a los ancianos ${ }^{31}$. Se cree que la sensibilización tiene lugar en el ambiente laboral, y por tanto se debería aplicar como medida preventiva primaria la sustitución de los jabones y detergentes por productos libres de fragancias. ${ }^{31,32}$

Los datos hallados con respecto a la parafenilendiamina, con una relevancia del $66,7 \%$, se explican porque este compuesto, que suele encontrarse en tintes del pelo y en el cuero, podría sensibilizar a este grupo por reacción cruzada con los componentes PPD de las gomas. De hecho, en nuestra muestra existe una ex-peluquera con sensibilización previa a este compuesto que vuelve a presentar síntomas en su nuevo trabajo como auxiliar. Del resto de sensibilizantes tampoco se pueden extraer grandes implicaciones, ya que al ser una muestra reducida y sólo encontrarse una positividad, aunque ésta sea clínicamente relevante, no son valorables. Lo que sí podría llamar la atención es que el formaldehído, que es considerado de relevancia ocupacional en trabajadores sanitarios en un $60 \%$, no obtuvo ninguna positividad en nuestro estudio. Es usado como desinfectante, preservante y esterilizante, y habitualmente se considera como posible sensibilizante en este grupo profesional ${ }^{30}$. Quizá pueda deberse a que en las residencias de ancianos no se utilizan con tanta asiduidad productos tan agresivos como en otras especialidades, como las quirúrgicas o la anatomía patológica.

Otro dato que hay que destacar son los resultados concernientes al uso de guantes. El 100\% de los pacientes refiere usar guantes como medida protectora, siendo los más utilizados los de látex-goma (85\%), seguidos por los de vinilo (34,15\%), algodón (21,95\%) y plástico (9,75\%). El 24,39\% (10 casos) relaciona su patología con el uso de dichos guantes, pero entre los diagnósticos sólo se observaron 3 casos de hipersensibilidad inmediata al látex (1 de ellos sin clínica).

Resulta sorprendente el alto porcentaje de uso de guantes y la poca prevalencia de hipersensibilidad o urticaria al látex, que no coincide con los datos encontrados habitualmente en los profesionales sanitarios, los cuales son considerados como el grupo ocupacional más afectado con respecto al resto, con una prevalencia de hipersensibilidad al látex en algunos trabajos de hasta un 16,7\% y de urticaria de contacto al látex de hasta un $12,8 \%{ }^{33}$. Se podría explicar porque los cuidadores de ancianos en realidad no usaran los guantes de goma con tanta asiduidad y los utilizaran sólo puntualmente para algún tipo de trabajo concreto, o durante poco tiempo a lo largo del día.

Por último, podríamos destacar que aunque existen multitud de estudios de las dermatosis profesionales en trabajadores de trabajo húmedo $\mathrm{y}$ profesionales sanitarios, son pocos los estudios existentes en la literatura que, como el nuestro, se centren en este colectivo profesional en particular, y por tanto se pueden establecer pocas comparaciones. En algunos países orientales se han llevado a cabo estudios en residencias de ancianos para establecer la prevalencia y características de enfermedades de la piel entre el personal de las mismas. El estudio se ha centrado en determinados centros y se ha incluido tanto personal sanitario como no sanitario. Los resultados no son coincidentes entre sí, ni con nuestro grupo, pero en todo caso demuestran una alta prevalencia de enfermedades de piel en estos trabajadores. En Taiwan Sur se evaluaron 11 residencias (84 trabajadores), siendo las enfermedades más frecuentemente diagnosticadas las infecciones fúngicas $(21,4 \%)$, la xerosis $(13,3 \%)$, la escabiosis $(10,7 \%)$ y finalmente la dermatitis ocupacional (8\%), con una prevalencia de esta última menor que la de otros estudios ${ }^{15}$. Los datos obtenidos en los estudios core- 
ano (1 residencia de 91 trabajadores $)^{14}$ y japonés (1 residencia de 89 trabajadores) 16 muestran como patología más frecuente la dermatitis de contacto, pero con una prevalencia muy baja en el primero de ellos (4,8\%) a diferencia del $12,7 \%$ más aproximado a otros estudios del segundo. Por último, un cuarto estudio compara la patología cutánea de ya sólo los trabajadores sanitarios de dos residencias en Japón y Corea $^{17}$. Demuestran que al igual que en nuestro grupo, la mayoría de los participantes eran mujeres (96,1\%) y que en el grupo japonés toda la patología cutánea era más frecuente que en el coreano, explicando este dato por el hecho de que la prevalencia de dermatitis atópica es más alta en Japón.

Se trata de estudios que aunque pueden no ser representativos de la situación global porque se limitan a grupos pequeños, documentan la importancia de esta patología, llaman la atención sobre la necesidad de estudios futuros con el fin de determinar factores de riesgo y contribuyen a establecer medidas preventivas eficaces, de gran importancia, ya que las dermatosis profesionales causan multitud de problemas socio-sanitarios que abarcan desde depresión a bajas laborales frecuentes o prolongadas, así como cambio o abandono del trabajo.

Dada la importancia de las condiciones endógenas, que facilitarían el desarrollo de dermatitis en pacientes predispuestos, una de las estrategias de prevención de las dermatosis profesionales más importante sería la del consejo médico previo a la elección de la carrera profesional o a la contratación. De hecho, este estudio clínico previo a la elección de trabajo es ya uno de los más extendidos y apoyados, y buscaría desalentar a los pacientes atópicos de desempeñar trabajos húmedos ${ }^{19,21,27}$; informar de los peligros que pueden conllevar ciertas profesiones de riesgo, así como aconsejar y formar a los trabajadores para que se protejan ${ }^{7,25}$. En Dinamarca ya está empezando a tener sus efectos positivos esta medida ${ }^{19}$ y podría ser bastante beneficioso el aplicarlo en el colectivo de cuidadores de ancianos.
También se debería llevar a cabo programas de educación e instrucción del cuidado de la piel en los trabajadores, para así disminuir la exposición a irritantes. Se debe fomentar el lavado de manos sólo cuando sea necesario, el uso de detergentes suaves y libres de fragancias, junto con productos tópicos protectores. Ya se ha demostrado su eficacia en un estudio realizado en enfermeras de geriatría. ${ }^{19,21,24,31,32}$

Por último, cabe destacar que pese al incremento de las incidencias de alergia al látex relacionado con el uso de guantes, estos son el mejor método para la prevención de dermatitis causadas por el trabajo húmedo, aunque debe potenciarse el uso de aquellos compuestos por materiales sustitutos del látex y su utilización sólo cuando es estrictamente necesario $23,27,28$. Todas estas medidas podrían llevarse a cabo en el colectivo de cuidadores de ancianos para disminuir la incidencia de dermatosis.

Como conclusión, cabe destacar que el colectivo de cuidadores de ancianos es un grupo en aumento y con gran importancia social. Como profesión con unas labores extensas y mal definidas que implican trabajo húmedo, se debe considerar de riesgo para el desarrollo de dermatosis profesionales. En nuestro estudio, basado en los cada vez más frecuentes auxiliares de geriatría que consultan por presentar problemas cutáneos, destaca la alta frecuencia del eczema en manos de origen endógeno, que sería explicada por el hecho de que la irritación provocada por el trabajo húmedo y la oclusión desencadenaría lesiones en una persona con un fondo atópico no manifestado previamente. Aunque puesta de manifiesto la problemática, nuevos estudios son necesarios para caracterizar los riesgos concretos de este grupo y llevar a cabo las medidas preventivas pertinentes. 


\section{BIBLIOGRAFÍA}

1. Kralj N, Michaelis M, Hofmann F. Prevalence of Skin Damage in Health Care Workers. J Occup Health 2000; 42:38-43.

2. Diepgen TL. Occupational skin-disease data in Europe. Int Arch Occup Environ Health 2003; 331-338.

3. Lushniak BD. The importance of occupational skin diseases in the United States. Int Arch Occup Environ Health 2003; 76: 325-330.

4. Belsito DV. Occupational contact dermatitis: Etiology, prevalence, and resultant impairment/disability. J Am Acad Dermatol 2005; 53: 303-313.

5. Diepgen T.L., Coenraads P.J. The epidemiology of occupational contact dermatitis. Int Arch Occup Environ Health 1999; 72:496-506.

6. Lushniak DB. Occupational contact dermatitis. Dermatol Ther 2004; 17(3): 272-7.

7. Meding B. Differences between the sexes with regard to work-related skin disease. Contact Dermatitis 2000; 43: 65-71.

8. Conde- Salazar Gómez L, Ancona-Alayón A. Dermatología Profesional. Edición 2004. Aula Médica. Páginas 41-48, 375-391.

9. Belsito DV. Healthcare Workers. En: Kanerva L, Elsner P, Wahlberg JE et al. Handbook of occupational dermatology. $1^{a}$ ed. Berlín: Springer- Verlag, 2000: 969973.

10. Fischer T. House Workers. En: Kanerva L, Elsner P, Wahlberg JE et al. Handbook of occupational dermatology. $1^{\mathrm{a}}$ ed. Berlín: Springer- Verlag, 2000: 978-980.

11. Lorenzo Carrascosa L. Consecuencias del envejecimiento de la población: El futuro de las pensiones. INE (Instituto Nacional de Estadística). http//www. ine.es consultado mayo 2006.

12. Las personas mayores en España. Observatorio de Personas Mayores. Informe 2004. Volumen 1-2. IMSERSO. Ministerio de Trabajo y Asuntos Sociales. Madrid.

13. INE. Censo de población y vivienda. Proyección de la población calculada a partir del Censo de 2001.

14. Smith DR, Choi JW, Yu DS, Ki M, Oh CH, Yamagata Z. Skin disease Among Staff in a large Korean Nursing Home. Tohoku J Exp Med. 2002; 198(3): 175-80.
15. Smith DR, Guo YLL, Lee YL, Hsieh FS, Chang SJ, Sheu HM. Prevalence of Skin Disease among Nursing Home Staff in Southern Taiwan. Ind Health 2002; 40: 5458.

16. Smith DR, Kubo H, Tang S, Yamagata Z. Skin Disease among Staff in a Japanese Nursing Home. J Occup Health 2003; 45(1): 60-2.

17. Smith DR, Choi JW, Yu DS, Ki M, Kubo H, Yamagata Z. A comparison of skin disease among nursing-home health care workers in Japan and Korea. Safety Science Monitor 2004; 1(8):1-4.

18. Nielsen J. The occurrence and course of skin symptoms on the hands among female cleaners. Contact Dermatitis 1996; 34: 284-291.

19. Skoet R, Olsen J, Mathiesen B, Iversen L, Johansen JD, Agner T. A survey of occupational hand eczema in Denmark. Contact Dermatitis 2004; 51: 159-166.

20. Löffler H, Dickel H, Bruckner T, Effendy I, Happle R. Skin changes in geriatric nurses prior to training heralding a particular risk of hand dermatitis. Eur J Dermatol 2002; 12(5): 452-454.

21. Dickel H, Kuss O, Schmidt A, Kretz J, Diepgen TL. Importance of Irritant Contact Dermatitis in Occupational Skin Disease. Am J Clin Dermatol 2002; 3(4): 238289.

22. Dickel H, Kuss O, Blesius CR, Schmidt A, Diepgen TL. Occupational skin diseases in Northern Bavaria between 1990 and 1999: a population-based study. Br J Dermatol 2001; 145: 453-462.

23. Jungbauer FHW, Lensen GJ, Groothoff JW, Coenraads PJ. Exposure of the hands to wet work in nurses. Contact Dermatitis 2004, 50:225-229.

24. Schürer NY, Klippel U, Schwanitz HJ. Secondary individual prevention of hand dermatitis in geriatric nurses. Int Arch Occup Environ Health 2005; 78: 149-157.

25. Dickel H, Bruckner TM, Schmidt A, Diepgen TL. Impact of Atopic Skin Diathesis on Occupational Skin Disease Incidence in a Working Population. J Invest Dermatol 2003; 121: 37-40.

26. Gawkrodger DJ, Lloyd MH, Hunter JAA. Occupational skin disease in hospital cleaning and kitchen workers. . Contact Dermatitis 1986; 15: 132-135. 
27. Nettis E, Colanardi MC, Soccio AL, Ferrannini A, Tursi A. Occupational irritant and allergic contact dermatitis among healthcare workers. Contact Dermatitis 2002; 46: 101-107.

28. Jungbauer FHW, Steenstra FB, Groothoff JW, Coenraads PJ. Characteristics of wet work in nurses. Int Arch Occup Environ Health 2005; 78: 248-251.

29. Strauss RM, Gawkrodger DJ. Occupational contact dermatitis in nurses with hand eczema. Contact Dermatitis 2001; 44: 293-296.

30. Dickel H, Kuss O, Schmidt A, Diepgen TL. Occupational relevance of positive standard patch-test results in employed persons with an initial report of an occupa- tional skin disease. Int Arch Occup Environ Health 2002; 75: 423-434.

31. Uter W, Schnuch A, Geier J, Pfahlberg A, Gefeller O. Association between occupational and contact allergy to the fragrance mix: a multifactorial analysis of national surveillance data. Occup Environ Med 2001; 58: 392-398.

32. Buckley DA, Rycroft RJG, White R, McFadden JP. Fragance as an occupational allergen. Occup Med 2002; 52: 13-16.

33. Valks R, Conde-Salazar L, Cuevas M. Allergic contact urticaria from natural rubber latex in healthcare and non-healthcare workers. Contact Dermatitis 2004; 50: 222-224. 